\title{
MULTI-COMPONENT EXERCISE WITH HIGH-INTENSITY, FREE-WEIGHT, FUNCTIONAL RESISTANCE TRAINING IN PRE-FRAIL FEMALES: A QUASI-EXPERIMENTAL, PILOT STUDY
}

\author{
N.W. BRAY ${ }^{1}$, G.J. JONES ${ }^{1}$, K.L. RUSH ${ }^{2}$, C.A. JONES ${ }^{3}$, J.M. JAKOBI ${ }^{1}$ \\ 1. School of Health and Exercise Sciences, Faculty of Health and Social Development, University of British Columbia Okanagan, Kelowna, British Columbia, Canada; 2. School of

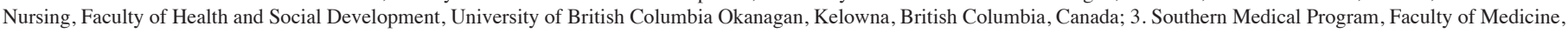 \\ University of British Columbia Okanagan, Kelowna, British Columbia, Canada. \\ Corresponding author: Jennifer M. Jakobi, School of Health and Exercise Sciences, Faculty of Health and Social Development, University of British Columbia Okanagan, Kelowna, \\ British Columbia, Canada, V1V 1V7, jennifer.jakobi@ubc.ca
}

\begin{abstract}
Background: No study has performed an exercise intervention that included high-intensity, freeweight, functional resistance training, and assessed frailty status as an inclusion criteria and outcome measure via original, standardized tools, in pre-frail females. Objectives: Determine if the intervention strategy is not only feasible and safe, but can also improve frailty status, functional task performance, and muscle strength. Design: Pilot, quasi-experimental. Setting: Community. Participants: 20 older-adults with pre-frailty characteristics. Intervention: 12-weeks (3 days/week, 45-60 minutes/session) of multi-component exercise, inclusive of aerobic, resistance, balance and flexibility exercises. The crux of the program was balance and resistance exercises, the latter utilized high-intensity, free-weight, functional resistance training. The control group maintained their usual care. Measurements: 1) Feasibility and safety (dropout, adherence, and adverse event); 2) Frailty (Frailty Phenotype, Clinical Frailty Scale, and gait speed); 3) Functional task performance (grip strength and sit-to-stand time); and 4) Isometric and isotonic strength of the knee extensors and elbow flexors. Results: No participants dropped out of the intervention or experienced an adverse event, and adherence averaged $88.3 \%$. The exercise group became less frail, whereas the control group became more frail. There was a significant within-group improvement in exercise participants gait speed $(\mathrm{p} \leq 0.01,+0.24 \mathrm{~m} / \mathrm{sec})$, grip strength $(\mathrm{p} \leq 0.01,+3.9 \mathrm{~kg})$, and sit-to-stand time ( $\mathrm{p} \leq 0.01,-5.0 \mathrm{sec})$. There was a significant within-group improvement in exercise participants knee extension isometric torque $(\mathrm{p} \leq 0.05,+7.4 \mathrm{Nm})$ and isotonic velocity $(\mathrm{p}=\leq 0.01,+37.5 \% \mathrm{sec})$. Elbow flexion isotonic velocity significantly declined within the control group $(\mathrm{p} \leq 0.01,-20.2 \% \mathrm{sec})$ and demonstrated a significant between-group difference ( $\mathrm{p} \leq 0.05,40.73 \% \mathrm{sec})$ post-intervention. Conclusions: The intervention strategy appears to be feasible and safe, and may also improve frailty status, functional task performance, and muscle strength. These results help calculate effect size for a future randomized controlled trial.
\end{abstract}

Key words: Older age, resistance training, muscle strength, quality of life, females.

\section{Introduction}

Exercise is considered a suitable therapy to reverse frailty (1-3) but it is difficult to determine which characteristics of an exercise program are most effective $(4,5)$. Most exercise intervention studies do not adequately assess the level of frailty (4), convoluting the understanding of positive adaptation and limiting the potential to apply spectrum specific exercise recommendations suggested in a recent literature review (1).

Frailty is also a dynamic process where transitions are subtle $(6,7)$, bidirectional $(8)$, and sex-dependent; females experience frailty differently than males, succumbing to the syndrome earlier yet living longer $(9,10)$. Older females also represent a larger percentage of the aging demographic (11), and pre-frail females outnumber those that are frail $(12,13)$. Thus, there is considerable need to identify exercise interventions that would improve frailty status in this large and growing cohort;

A scoping review identified only 15 exercise studies that used a frailty identification tool to classify participants' Received August 12, 2019

Accepted for publication December 6, 2019 frailty status at the onset and conclusion of an intervention (14). However, only five studies (15-19) included a clearly defined pre-frail sample population and only one focused exclusively on females, which were identified via a modified frailty assessment tool (17). These studies generally utilized higher-repetition, low-intensity, single-joint resistance training exercises. To-date, no study has utilized validated frailty identification tools as both an inclusion criteria and outcome measure to assess an exercise intervention that incorporates high-intensity, free-weight, functional resistance training, exclusively in pre-frail females. Despite a lack of evidence supporting such claim, this may be due to the commonly held belief that such resistance training exercises are unsafe (20).

We performed a pilot study to determine if a multicomponent exercise (MCE) program, inclusive of high intensity, free-weight, functional resistance training, is not only feasible and safe, but can improve frailty status, functional task performance, and measures of strength in pre-frail females. It was hypothesized that the exercise group (EX) would improve 
Figure 1

Overview of the multi-component exercise (MCE) intervention and assessments. Similar to resistance training, participants began balance training at different levels and progressed based upon individual ability. The balance training was progressed when the upper limit for time or repetition range was achieved without loss of balance. Training sessions concluded with a flexibility training, utilizing just a kneeling hip flexor flexibility exercise (30). Participants started by stretching for 15 seconds (sec) per leg, and added $15 \mathrm{sec}$ each week, up to a maximum of $60 \mathrm{sec}$, for a total time of $2 \mathrm{~min}$

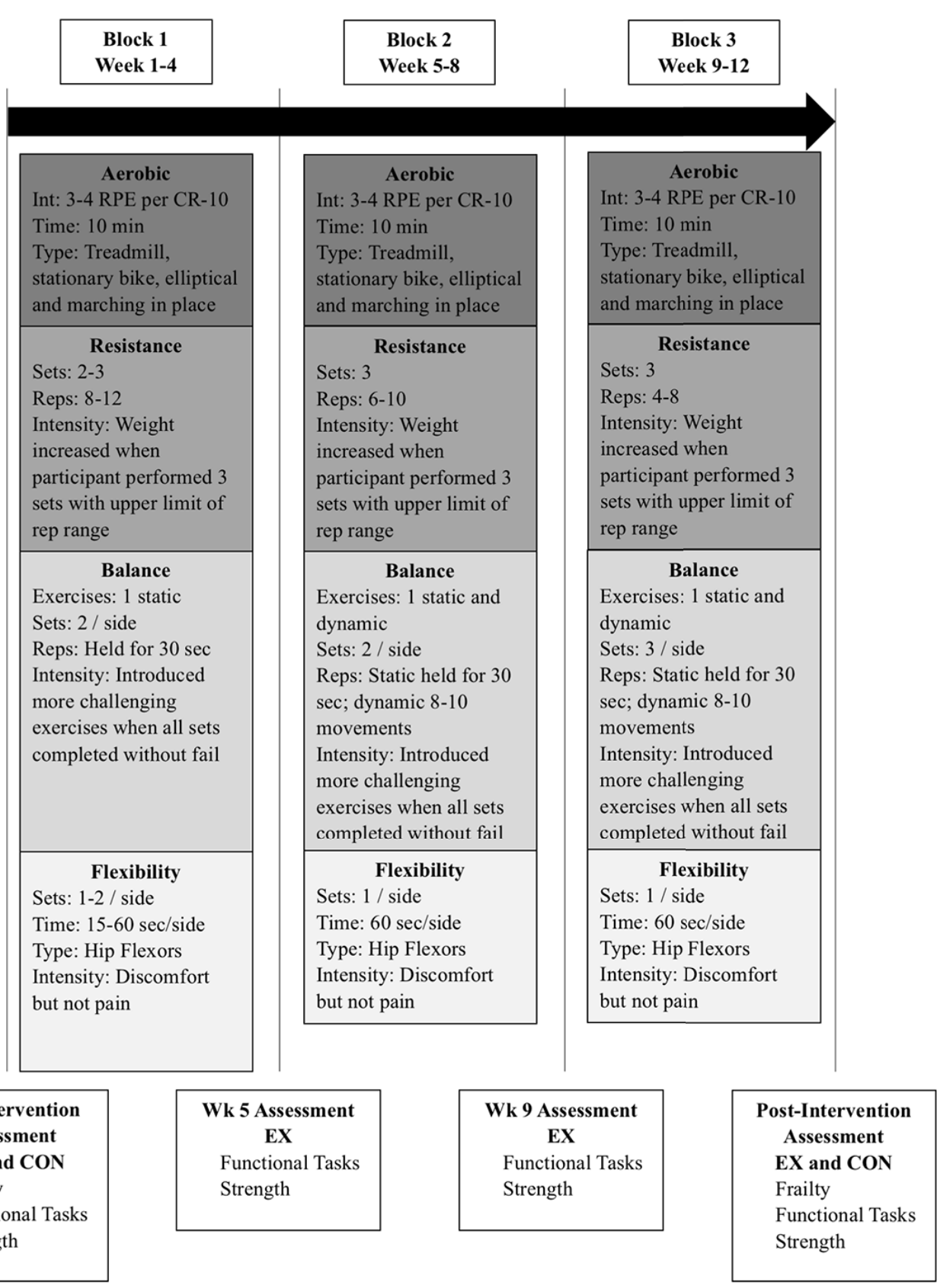

Int $=$ intensity $; \mathrm{RPE}=$ rating of perceived exertion; $\min =$ minutes $;$ rep $(\mathrm{s})=$ repetition $(\mathrm{s}) ; \mathrm{sec}=$ seconds $; \mathrm{EX}=\mathrm{Exercise} ; \mathrm{CON}=\mathrm{Control} ; \mathrm{wk}=\mathrm{week}$ 


\section{THE JOURNAL OF FRAILTY \& AGING}

frailty status, functional task performance, and muscle strength, in comparison to a control group (CON).

\section{Methods}

\section{Experimental Overview}

EX participants completed a 12-week exercise intervention (3-days/week, 45-60 min/session). The CON maintained their normal routine for the same duration. Participants of both groups were assessed for frailty status, functional task performance, and muscle strength at week 0 (pre-intervention) and 13 (post-intervention), and the latter two measures were repeated at weeks 5 and 9 for the EX (Figure 1).

\section{Subjects}

Participants were recruited from the community. Inclusion criteria consisted of: 1) Females $\geq 65$ years of age; 2) A Montreal Cognitive Assessment (21) score $\geq$ adjusted normative values (22);3) No contraindications to exercise, as determined by the Physical Activity Readiness-Questionnaire Plus; 4) No major injuries/surgeries to the dominant arm or leg in the last six months; 5) Fluent in English; and 6) A pre-frail frailty status.

Initial recruitment found 53 older adults interested but only 21 agreed to complete the pre-intervention assessment, with one being excluded. As a quasi-experimental study, participants were randomized to the EX and CON based upon their perceived availability to participate in exercise. Current interventions utilizing exercise require $80 \%$ adherence (23). As a result, 9/20 participants were placed in the EX.

All participants read and signed a letter of informed consent. Ethical approval was granted by the institutional Research Ethics Board (H16-00712). All experimental procedures involved in the study conformed to the declaration of Helsinki.

\section{Multi-Component Exercise (MCE)}

Kinesiology students led each session. Details of the aerobic warm-up, as well as the balance exercises and flexibility cooldown are within Figure 1. Resistance training was divided into three blocks of four weeks; each new block decreased the number of repetitions to be completed and subsequently increased the resistance, thus, transitioning from training muscular endurance to strength. Within each block, the resistance was also increased when participants reached the upper limit of the repetition range for all sets. Rest periods between sets ranged from 1-3 minutes. To aid progressive overload, participants disclosed their rating of perceived exertion after the last set of every exercise. Details about the training principles can be found in our companion article, "Practical Implications for Strength and Conditioning of Older Pre-Frail Females" (24).

Four free-weight resistance exercises were selected: 1) Squat; 2) Deadlift; 3) Bench Press; and 4) Leg Press. Exercises 1-3 replicate functional movements, such as standing from a toilet, opening a door, and picking up groceries from the ground; these exercises utilized dumbbells (Hex Dumbbell, Northern Lights Inc, Cornwall, ON) and/or barbells (The Bella 2.0 - Females's Bar, Rogue Fitness; Columbus, $\mathrm{OH}$ ) with weighted plates (Virgin Rubber Grip Olympic Plates, Element Fitness; Latvia). The fourth exercise supplemented the Squat exercise but was less technically demanding, and utilized an incline leg press machine (TuffStuff PPL-960 45 Leg Press, TuffStuff Fitness Equipment, Chino, CA). Each session started with the squat or bench press and finished with the deadlift or inclined leg press.

\section{Experimental Assessments}

Feasibility and Safety: Participant dropout and adverse events were recorded in an ongoing electrical document log. Adherence rates were maintained in an ongoing paper log that participants were required to sign upon arrival to each intervention session.

Frailty Status: Participants were included in this study if they were classified as pre-frail according to guidelines in at least one of the following three tools:

1) 1-2 on the frailty phenotype (FP) (25)

2) 4-6 on the clinical frailty scale (CFS) (26)

3) Gait speed (GS) of $\geq 1.0-<1.5 \mathrm{~m} / \mathrm{sec}$ indicated pre-frailty (27)

Previous research suggests that several frailty tools may provide a more reliable measure of frailty status, and that the FP and CFS do not always provide the same classification $(9,28)$. Therefore, GS was used as a third frailty criterion.

Functional Task Performance: GS was assessed across 8 meters $(\mathrm{m})$, excluding acceleration $(2 \mathrm{~m})$ and deceleration $(2 \mathrm{~m})$ zones, at a self-selected walking speed that was considered normal. The GS test was completed twice and the fastest trial was used for data analysis. Handgrip strength was measured while standing with a dynamometer (Baseline Smedley, Fabrication Enterprises Incorporated, White Plains, NY) held at arm's length and slightly abducted. The dynamometer was squeezed as hard as possible for three seconds, and completed twice for each hand. The order of grip testing was randomized and trials occurred between gait tests. The highest score was used for both functional task performance and FP. The sitto-stand (STS) task was adopted from the short physical performance battery (29).

Muscle Strength Performance: The Biodex Dynamometer System 4 Pro (Biodex Medical Systems Incorporated, Shirley, $\mathrm{NY}$ ) was used to assess peak torque/velocity of isometric/ isotonic knee extension (KE) and elbow flexion (EF). For KE and EF, participants were seated with their hips flexed to $\sim 100^{\circ}$. Restraining straps crossed the chest and opposite hip, and for $\mathrm{KE}$, a third was secured across the thigh of the tested leg. For $\mathrm{KE}$, the lateral femoral condyle of the dominant leg was aligned with the dynamometer center of rotation. To account for the effect of gravity, the weight of the limb was calculated by the 
Figure 2

Changes in frailty status across all three frailty identification tools: A) Frailty Phenotype (FP), n = 16, B) Clinical Frailty Scale (CFS), $n=16$ and C) gait speed (GS), $n=16$. Pre-frailty thresholds indicated by the vertical broken lines; 1-2 on the FP, or 4-6 on the CFS, or a GS between $\geq 1.0$ to $<1.5$ meters per second $(\mathrm{m} / \mathrm{sec})$. The order of participants is consistent between each figure, in order to identify individual frailty scores across tools, as well as the change between time points. The arrow head indicates the direction and magnitude of change (right = negative or more frail; left = positive or less frail) from pre to post-intervention . Pre-intervention, nine, three, and thirteen participants were classified as pre-frail according to the FP, CFS, and GS, respectively. Pre-intervention, one participant was considered pre-frail according to all three tools, while seven participants were classified as pre-frail according to two tools. $\mathbf{\square}=$ exercise $(\mathrm{EX}), \boldsymbol{0}=\operatorname{control}(\mathrm{CON})$ group

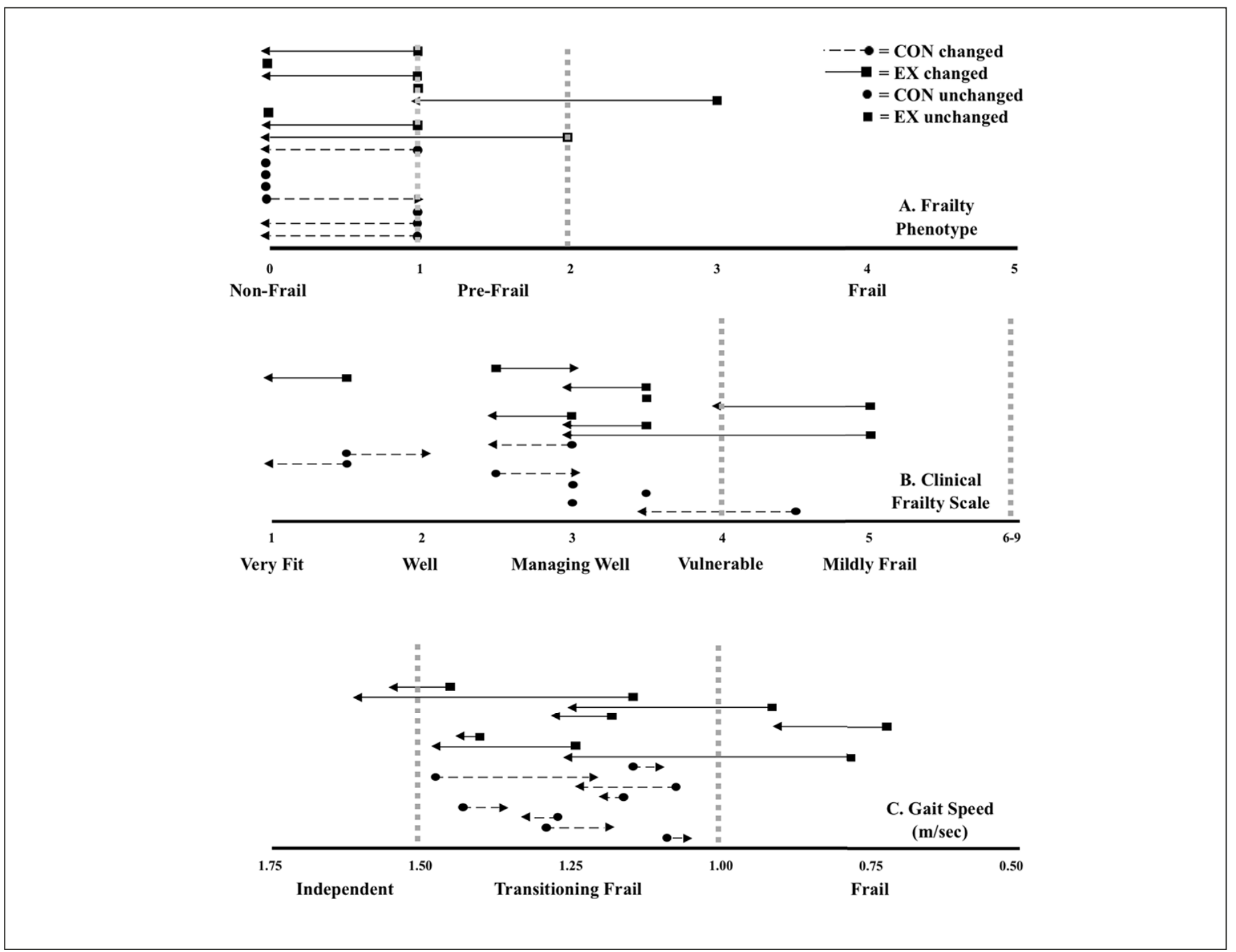

Biodex with the knee extended to $160^{\circ}$. For EF, the medial epicondyle of the elbow was aligned with the dynamometer center of rotation. The shoulder was slightly forward flexed $\left(10-15^{\circ}\right)$ and abducted. Positioning was measured, recorded, and replicated for all sessions.

Isometric $\mathrm{KE}$ and $\mathrm{EF}$ contractions were executed at a joint angle of $90^{\circ}$. Isotonic $\mathrm{KE}$ was also initiated from $90^{\circ}$ but $\mathrm{EF}$ isotonic contractions were initiated from $160^{\circ}$. Range of motion of isotonic contractions was $90-160^{\circ}$ for $\mathrm{KE}$ and the inverse for $\mathrm{EF}$. Isotonic resistance was $20 \%$ of the peak torque from the strongest isometric contraction of the pre-intervention assessment. Participants completed five isometric and isotonic contractions for both $\mathrm{KE}$ and $\mathrm{EF}$, with two minutes of rest between contractions. All contractions were recorded and the highest value was used for data analysis. The analogue signal was sampled at $2,000 \mathrm{~Hz}$ and stored for offline analysis using the Biodex software. 


\section{THE JOURNAL OF FRAILTY \& AGING}

\section{Statistical Analysis}

Pre-intervention characteristics were compared using an independent sample t-test. A sample size calculation for a 2-tailed study design advised a minimum sample size of six subjects per group to attain a statistical power of 0.80 for the KE strength variable. A two-way mixed ANOVA of time by group assessed all outcomes. A one-way ANOVA was used to evaluate any significant interactions and main effects. The EX was also examined over time using a one-way repeated measures ANOVA, with a Bonferroni post-hoc test. Sphericity was assessed (Mauchly's) and a Greenhouse-Geisser correction applied if violated $(\mathrm{p}<0.05)$. Data that violated normality and homogeneity of variances was transformed using the function Log 10. Statistical significance was $p \leq 0.05$. All values are reported as mean \pm standard deviation (SPSS Statistics V.24, IBM Canada Ltd. Markham, Ontario).

\section{Results}

Characteristics: Participant characteristics were similar between groups (Table 1). Three CON participants were removed from the analysis because they were unable to complete the post-intervention assessment at the required date due to a fall-related injury unrelated to the study $(n=2)$ or illness $(n=1)$. No EX participants dropped out or experienced an adverse event during the intervention, and intervention adherence averaged $88.3 \%$. One EX participant completed $<65 \%$ of exercise sessions (seasonal flu) and was subsequently removed from the final analysis. A shoulder injury, unrelated to the exercise program, precluded one EX participant from completing the grip strength, STS, and EF measures at week 13.

Table 1

Participant characteristics (mean $\pm \mathrm{SD})$

\begin{tabular}{lcc}
\hline Characteristics & CON & EX \\
\hline & $\mathrm{n}=8$ & $\mathrm{n}=8$ \\
Age (years) & $72.4 \pm 5.4$ & $72.9 \pm 4.8$ \\
Height $(\mathrm{cm})$ & $156.4 \pm 7.7$ & $162.5 \pm 4.0$ \\
Weight $(\mathrm{kg})$ & $67.1 \pm 11.0$ & $75.8 \pm 23.3$ \\
BMI $\left(\mathrm{kg} / \mathrm{m}^{2}\right)$ & $27.4 \pm 4.0$ & $28.6 \pm 8.5$ \\
MoCA & $25.6 \pm 2.6$ & $25.0 \pm 3.0$ \\
Comorbidities per Participant & $1.9 \pm 1.5$ & $2.1 \pm 1.2$ \\
\hline
\end{tabular}

$\mathrm{SD}=$ standard deviation $; \mathrm{CON}=$ control; $\mathrm{EX}=$ exercise; $\mathrm{BMI}=$ body mass index; MoCA = Montreal Cognitive Assessment; $\mathrm{cm}=$ centimeters; $\mathrm{kg}=$ kilograms; $\mathrm{kg} / \mathrm{m} 2$ = kilograms per meter squared.

Frailty: There was a time main effect for the FP $(\mathrm{F}(1,14)$ $=8.5, \mathrm{p} \leq 0.01$, partial $\eta 2=0.4)$ and $\operatorname{CFS}(\mathrm{F}(1,14)=4.8, \mathrm{p}$ $\leq 0.05$, partial $\eta 2=0.3)$ but no group main effect $(\mathrm{F}(1,14)$ $=1.423, \mathrm{p}=0.25$, partial $\eta 2=0.09)$. Following the 12 -week intervention, five and six participants of the EX were less frail according to the FP and CFS, respectively. Gait speed was faster for all EX participants. The CON became more frail according to the FP $(n=1), \operatorname{CFS}(n=2)$, and GS $(n=8)$, respectively (Figure 2).

\section{Figure 3}

Control $(\mathrm{CON})$ and Exercise $(\mathrm{EX})$ group results for functional task measures

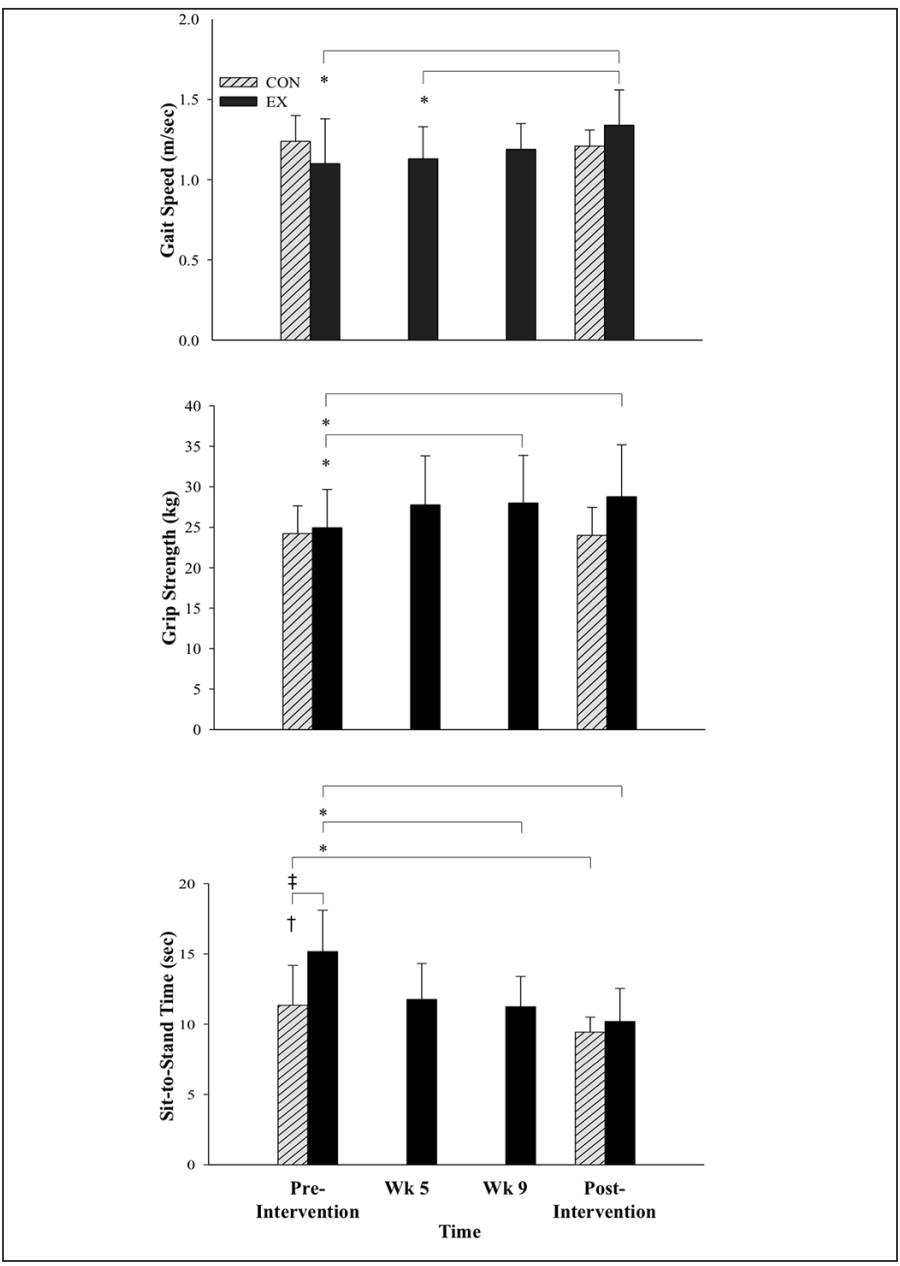

Gait speed, $\mathrm{n}=16$, grip strength, $\mathrm{n}=15$, and sit-to-stand time, $\mathrm{n}=15$ pre and postintervention. Intra-group assessments performed only on the EX at 5 and 9-weeks. *, significant difference within the EX over time; $\uparrow$, significant difference between groups; $\$$, significant difference within the CON over time. $\mathrm{m} / \mathrm{sec}=$ meters per second; $\mathrm{kg}=$ kilograms; $\mathrm{sec}=$ seconds; $\mathrm{wk}=$ week

Functional Task Performance: EX demonstrated improvements in post-intervention $\operatorname{GS}(\mathrm{F}(1,7)=15.2$, $\mathrm{p} \leq 0.01$, partial $\eta 2=0.7)$ and grip strength $(\mathrm{F}(1,6)=17.3$, $\mathrm{p} \leq 0.01$, partial $\eta 2=0.7$; Figure 3$)$. STS time $(\mathrm{F}(1,13)=4.8, \mathrm{p} \leq 0.05$, partial $\eta 2=0.3)$ in the CON was faster pre-intervention $(\mathrm{F}(1,13)=6.6, \mathrm{p}=0.02$, partial $\eta 2$ $=0.3)$ but no significant difference existed post-intervention $(\mathrm{F}(1,13)=0.7, \mathrm{p}=0.4$, partial $\eta 2=0.0)$ as a result of a significant improvement in the $\operatorname{EX~}(5.0 \mathrm{sec}, \mathrm{F}(1,6)=18.2$, $\mathrm{p} \leq 0.01$, partial $\eta 2=0.8$ ).

Muscle Strength Performance: KE isometric torque $(\mathrm{F}(1,7)$ $=5.9, \mathrm{p} \leq 0.05$, partial $\eta 2=0.5)$ and isotonic velocity $(\mathrm{F}(1,7)$ 
$=17.5, \mathrm{p}=\leq 0.01$, partial $\eta 2=0.7)$ improved in the EX postintervention (Figure 4). There was a significant interaction for EF isotonic velocity $(F(1,13)=14.8, p \leq 0.01$, partial $\eta 2=0.5)$ as the $\mathrm{CON}$ was slower post-intervention $\left(-20.2^{\circ} / \mathrm{sec}, \mathrm{F}(1,7)=\right.$ $21.7, \mathrm{p} \leq 0.01$, partial $\eta 2=0.8$ ).

\section{Figure 4}

Control (CON) and Exercise (EX) group results for strength measures

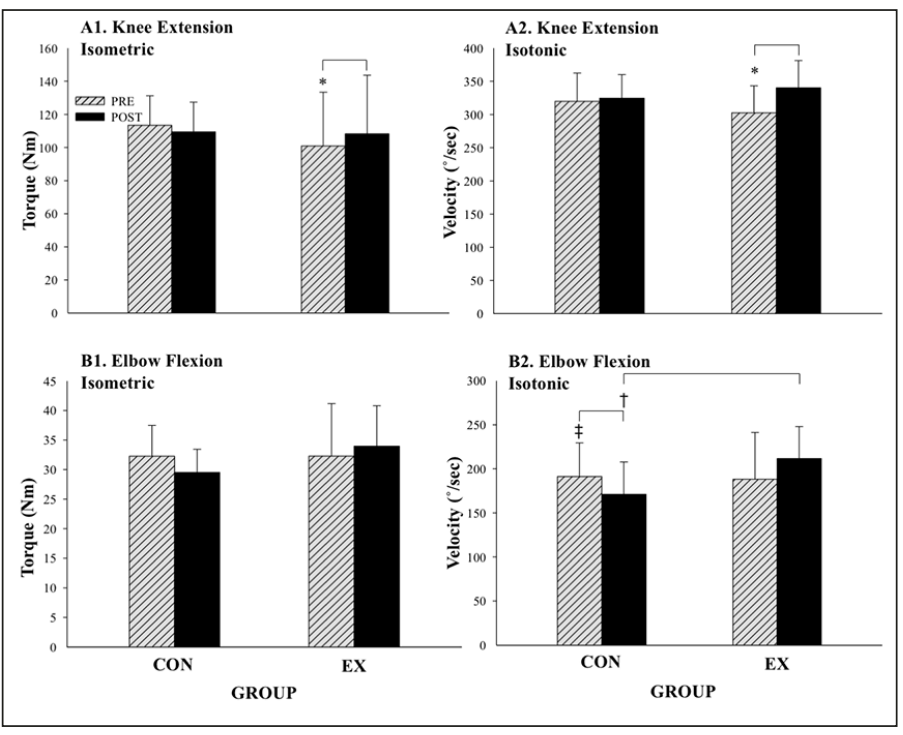

Knee extension (KE) isometric torque (A1), $\mathrm{n}=16, \mathrm{KE}$ isotonic velocity (A2), $\mathrm{n}=16$ Elbow flexion (EF) isometric torque (B1), $\mathrm{n}=15$ and $\mathrm{EF}$ isotonic velocity (B2), $\mathrm{n}=15$ pre and post-intervention. $*$, significant difference within the EX over time; $\dagger$, significant difference between groups; $\$$, significant difference within the CON over time. Pre $=$ preintervention; post $=$ post-intervention $; \mathrm{Nm}=$ newton meters $;{ }^{\circ} / \mathrm{sec}=$ degrees per second

\section{Discussion}

Findings suggest that this intervention strategy appears to not only be feasible and safe for pre-frail females, but it can also improve frailty status, functional task performance, and strength measures. Conversely, CON participants became more frail over the same time period, showed little change in GS, grip and $\mathrm{KE}$ strength, and suffered a significant decline in EF isotonic strength.

This is the first study to include high-intensity, free-weight, functional resistance training in pre-frail older females, assessed via non-modified frailty tools pre and post-intervention. The lack of adverse events suggest that this style of training is safe when supervised and programmed correctly. The high adherence rates and lack of dropout may indicate a high degree of enjoyment. Previous interventions in pre-frail older adults have generally utilized high-repetition, low-intensity, singlejoint resistance training exercises, which, despite a lack of evidence, may be attributed to the commonly held belief that the alternative is unsafe (20).

Although frailty status was inconsistent between tools, we believe that by utilizing a combination of tools, the sample is reflective of the pre-frail/vulnerable population under investigation. For example, at baseline, our participant with the fastest gait speed was also considered pre-frail according to the FP. Frailty is a dynamic syndrome, as indicated by the different levels of frailty described across most assessment tools $(25,26)$, and there is growing support for disassociating frail from prefrail $(1,5)$. Sex-differences also further complicate frailty $(9$, 10). Previous research have reported a wide-range of values for the percentage (21-48\%) of participants that have demonstrated an improvement in frailty status. Regardless of the tool, our percentage $(>63 \%)$ of participants that reversed frailty exceeds all other previous interventions that have included a pre-frail population, and measured frailty status pre and post-exercise intervention (15-19).

All measures of functional task performance consistently improved for the EX. Kwon and colleges (17) assessed both GS and grip strength, but only reported improvements in the latter $(2.3 \mathrm{~kg})$ for their intervention group, which was less than the $3.9 \mathrm{~kg}$ change observed in our EX. Improvements in functional task performance complement the observed changes in the Biodex measures.

The EX demonstrated significant improvement in $\mathrm{KE}$ isometric and isotonic strength post-intervention. Additionally, our EX maintained EF isotonic strength while the CON experienced a significant decline. Both Chan et al., (16) and $\mathrm{Ng}$ et al., (19) reported improvements in KE isometric strength in both their exercise and non-exercise groups postintervention, and therefore, exercise may not be responsible for improvement. Our study is the first to identify strength values for isotonic $\mathrm{KE}$, as well as isometric and isotonic EF in prefrail older females, as part of an exercise intervention that used standard frailty assessment tools as both an inclusion criteria and outcome measure.

Synergistically (24), our study findings suggest that this intense resistance training program is not only safe for pre-frail females, but necessary to make enhanced improvements in frailty status, functional task performance, and muscle strength. However, this study is not without limitation. The sample size was relatively small but strength measures were appropriately powered to observe statistically meaningful changes. Moreover, the small group size likely positively influenced participants' adherence to the program $(88.3 \%)$. Further work is needed to assess the factors that contribute to adherence in this population. Given the limited number of study personnel, the lead student investigator conducted the exercise training and was not blinded to group assignment but pre-intervention scores were unavailable at intra and post-intervention testing, and file names were coded during analysis. Despite the limitations, we believe the findings support undertaking a large randomized controlled trial.

\section{Conclusion}

MCE inclusive of high-intensity, free-weight, functional resistance training is feasible and safe in pre-frail older females. 


\section{THE JOURNAL OF FRAILTY \& AGING}

When compared to previous studies that have measured prefrailty status as both an inclusion criteria and outcome measure, it appears to lead to greater improvements in frailty status, functional task performance, and muscle strength. A larger randomized controlled trial is required to confirm our findings.

Funding: Partial funding for this study through the Canadian Institutes for Health Research (CIHR) Grant \# 385692. The sponsors had no role in the design and conduct of the study; in the collection, analysis, and interpretation of data; in the preparation of the manuscript; or in the review or approval of the manuscript.

Acknowledgments: We wish to acknowledge the support from; Flaman Fitness ${ }^{\mathrm{TM}}$ and the Okanagan Men's Shed Club for generously donating the exercise equipment, graduate students (Rowan Smart and Sam Kuzyk) and senior undergraduate students (Anup Dhaliwal, Brett Yungen, Savannah Frederick, Paul Cotton and Cydney Richardson), and all the participants involved in this study.

Ethics approval and consent: All participants read and signed a letter of informed consent. Ethical approval was granted by the institutional Research Ethics Board (H16-00712).

Availability of the data and materials: The original data and materials are available through the institutions open access graduate thesis repository https://open.library.ubc.ca/cIRcle/ collections/ubctheses/24/items/1.0353165

\section{Competing interests: None}

Trial Registration: This study was prospectively registered with ClincalTrials.gov (NCT02952443) on October 31, 2016.

Open Access: This article is distributed under the terms of the Creative Commons Attribution 4.0 International License (http://creativecommons.org/licenses/by/4.0/), which permits use, duplication, adaptation, distribution and reproduction in any medium or format, as long as you give appropriate credit to the original author(s) and the source, provide a link to the Creative Commons license and indicate if changes were made.

\section{References}

1. Bray NW, Smart RR, Jakobi JM, Jones GR. Exercise prescription to reverse frailty Appl Physiol Nutr Metab 2016;41(10):1112-16.

2. Theou O, Stathokostas L, Roland KP, et al. The effectiveness of exercise intervention for the management of frailty: a systematic review. J Aging Res 2011;2011:569194.

3. Jones GR, Jakobi JM. Launching a new initiative: Why is exercise and nutrition research important in addressing frailty? Appl Physiol Nutr Metab 2017;42(9):iii-iv.

4. de Labra C, Guimaraes-Pinheiro C, Maseda A, Lorenzo T, Millán-Calenti JC Effects of physical exercise interventions in frail older adults: a systematic review of randomized controlled trials. BMC Geriatr 2015;15:154.

5. Gine-Garriga M, Roque-Figuls M, Coll-Planas L, Sitja-Rabert M, Salva A. Physica Exercise Interventions for Improving Performance-Based Measures of Physical Function in Community-Dwelling, Frail Older Adults: A Systematic Review and Meta-Analysis. Arch Phys Med Rehabil 2014;95(4):753-69.e3.

6. Theou O, Jones GR, Vandervoort AA, Jakobi JM. Daily muscle activity and quiescence in non-frail, pre-frail, and frail older females. Exp Geronto
2010*45(12):909-17.

7. Bray NW, Doherty TJ, Montero-Odasso M. The Effect of High Dose Vitamin D3 on Physical Performance in Frail Older Adults. A Feasibility Study. J Frailty Aging 2018;7(3):155-61.

8. Roland KP, Theou O, Jakobi JM, Swan L, Jones GR. Exploring frailty: Community physical and occupational therapists' Perspectives. Phys Occup Ther Geriatr 2011;29(4):270-286.

9. Theou O, Brothers TD, Peña FG, Mitnitski A, Rockwood K. Identifying common characteristics of frailty across seven scales. J Am Geriatr Soc 2014;62(5):901-6.

10. Hubbard RE, Rockwood K. Frailty in older females. Maturitas 2011;69(3):203-7.

11. Statistics Canada. Canada's population estimates: Age and sex, July 1, 2015. 2015. https://www150.statcan.gc.ca/n1/daily-quotidien/150929/dq150929b-eng.htm. Accessed 01 August 2019

12. Bandeen-Roche K, Seplaki CL, Huang J, et al. Frailty in Older Adults: A Nationally Representative Profile in the United States. J Gerontol A Biol Sci Med Sci 2015;70(11):1427-34.

13. Hoover M, Rotermann M, Sanmartin C, Bernier J. Validation of an index to estimate the prevalence of frailty among community-dwelling seniors. Health Rep 2013;24(9):10-7

14. Puts MTE, Toubasi S, Andrew MK, et al. Interventions to prevent or reduce the leve of frailty in community-dwelling older adults: A scoping review of the literature and international policies. Age Ageing 2017;46(3):383-92.

15. Serra-Prat M, Sist X, Domenich R, et al. Effectiveness of an intervention to preven frailty in pre-frail community-dwelling older people consulting in primary care: A randomised controlled trial. Age Ageing 2017;46(3):401-7.

16. Chan D-CD, Tsou H-H, Yang R-S, et al. A pilot randomized controlled trial to improve geriatric frailty. BMC Geriatr 2012;12(1):58.

17. Kwon J, Yoshida Y, Yoshida H, Kim H, Suzuki T, Lee Y. Effects of a combined physical training and nutrition intervention on physical performance and healthrelated quality of life in prefrail older females living in the community: A randomized controlled trial. J Am Med Dir Assoc 2015;16(3):263.e1-e8.

18. Li C-M, Chen C-Y, Li C-Y, Wang W-D, Wu S-C. The effectiveness of comprehensive geriatric assessment intervention program for frailty in communitydwelling older people: a randomized, controlled trial. Arch Gerontol Geriatr 2010;50 Suppl 1:S39-42.

19. Ng TP, Feng L, Nyunt MSZ, et al. Nutritional, Physical, Cognitive, and Combination Interventions and Frailty Reversal among Older Adults: A Randomized Controlled Trial. Am J Med 2015;128(11):1225-36.

20. Watson SL, Weeks BK, Weis LJ, Horan SA, Beck BR. Heavy resistance training is safe and improves bone, function, and stature in postmenopausal women with low to very low bone mass: novel early findings from the LIFTMOR trial. Osteoporos Int 2015;26(12):2889-94.

21. Nasreddine ZS, Phillips NA, Bedirian V, et al. The Montreal Cognitive Assessment, MoCA: A Brief Screening Tool for Mild Cognitive Impairment. J Am Geriatr Soc 2005;53(4):695-9.

22. Malek-Ahmadi M, Powell JJ, Belden CM, et al. Age- and education-adjusted normative data for the Montreal Cognitive Assessment (MoCA) in older adults age 70-99. Neuropsychol Dev Cogn B Aging Neuropsychol Cogn 2015;22(6):755-61.

23. Montero-Odasso M, Almeida QJ, Burhan AM, et al. SYNERGIC TRIAL (SYNchronizing Exercises, Remedies in Gait and Cognition) a multi-Centre randomized controlled double blind trial to improve gait and cognition in mild cognitive impairment. BMC Geriatr. 2018;18(1):93.

24. Bray NW, Jones GR, Rush KL, Jones C A, Jakobi JM. Practical Implications for Strength and Conditioning of Older Pre-Frail Females. J Frailty Aging 2019; In Press.

25. Fried LP, Tangen CM, Walston J, et al. Frailty in Older Adults: Evidence for a Phenotype. J Gerontol A Biol Sci Med Sci 2001;56(3):M146-56.

26. Rockwood K, Song X, MacKnight C, et al. A global clinical measure of fitness and frailty in elderly people. CMAJ 2005;173(5):489-95.

27. Jones GR, Neubauer NA, O'Connor B, Jakobi JM. EMG Functional tasks recordings determines frailty phenotypes in males and females. Exp Gerontol 2016;77:12-8.

28. Lytwyn J, Stammers AN, Kehler DS, et al. The impact of frailty on functional survival in patients 1 year after cardiac surgery. J Thorac Cardiovasc Surg 2017;154(6):1990-9.

29. Guralnik JM, Simonsick EM, Ferrucci L, et al. A short physical performance battery assessing lower extremity function: association with self-reported disability and prediction of mortality and nursing home admission. J Gerontol 1994;49(2):M85-94.

30. Watt JR, Jackson K, Franz JR, et al. Effect of a supervised hip flexor stretching program on gait in elderly individuals. PM R 2011;3(4):324-9. 